\title{
Transient radiation myelopathy: spinal somatosensory evoked responses following incidental cord exposure during radiotherapy
}

\author{
B R F LECKY, N M F MURRAY, AND R J BERRY \\ From the Departments of Neurology and of Radiotherapy and Oncology, Middlesex Hospital, London \\ and the Department of Clinical Neurophysiology, The National Hospital, Queen Square, London
}

SUMMARY Serial spinal somatosensory evoked potentials were recorded in six patients undergoing radiotherapy involving incidental spinal radiation. Two patients developed transient radiation myelopathy. No abnormality was found in the somatosensory evoked potentials throughout the study.

The syndrome of transient radiation myelopathy was recognised by Boden ${ }^{1}$ and described in further detail by Jones. ${ }^{2}$ It is characterised by the early onset of Lhermitte's symptom or other peripheral tingling in the absence of other neurological symptoms or signs. None of the patients described subsequently developed neurological deficit and the condition, which is self-limiting, appears to be quite distinct from the later chronic progressive radiation myelopathy. Two of Jones's patients, dying approximately a year after treatment from their underlying malignant disease, were studied histopathologically. In one there was no abnormality of the cord and the other showed "comparatively insignificant" myelin pallor. Electron microscopy was not done.

Jones suggested that the condition was due to subclinical demyelination of the cervical cord white matter. The cumulative radiation doses responsible (30-71 Gy, 3000-7100 Rad) were felt to be insufficient to disrupt mature myelin lipoprotein, but it was suggested that they might disrupt the turnover and synthesis of new myelin. Radiation might cause inhibition of the myelin-forming oligodendrocytes; the latent interval before clinical sequelae might result from the normal longevity of previously synthesised myelin. Symptoms might then persist until biosynthesis of myelin was re-established.

Address for reprint requests: Dr BRF Lecky, Institute of Neurology, Queen Square, London WC1N 3BG.

Accepted 11 April 1980
There is experimental evidence indicating that oligodendrocytes are indeed affected early and severely in the irradiated cord. ${ }^{34}$ Further detailed studies of the irradiated rat cord by electron microscopy ${ }^{5}$ have demonstrated early breakdown of paranodal myelin and nodal widening as soon as two weeks after exposure. Sequential studies showed the subsequent appearance of thinly myelinated fibres, suggesting that the paranodal demyelination was followed by remyelination. The authors suggest that the former changes represented the pathological counterpart of human transient radiation myelopathy. Other changes were also noted, chiefly Wallerian-type degeneration increasing with time, and these were suggested to be the forerunners of delayed radionecrosis.

Spinal evoked responses (SEPs) are frequently abnormal in multiple sclerosis even in the absence of clinical evidence of cord involvement. ${ }^{6}$ Because there is the possibility of subclinical demyelination occuring after radiation, we hoped to detect abnormalities by the serial study of somatosensory evoked responses in patients undergoing radiotherapy.

\section{Patients and methods}

Six patients were studied (see table), all receiving incidental spinal irradiation during radiotherapy for neoplastic conditions. Except for patient 1, who received dexamethasone for superior vena caval obstruction, no patient received chemotherapy. 
Table Details of patients and treatment

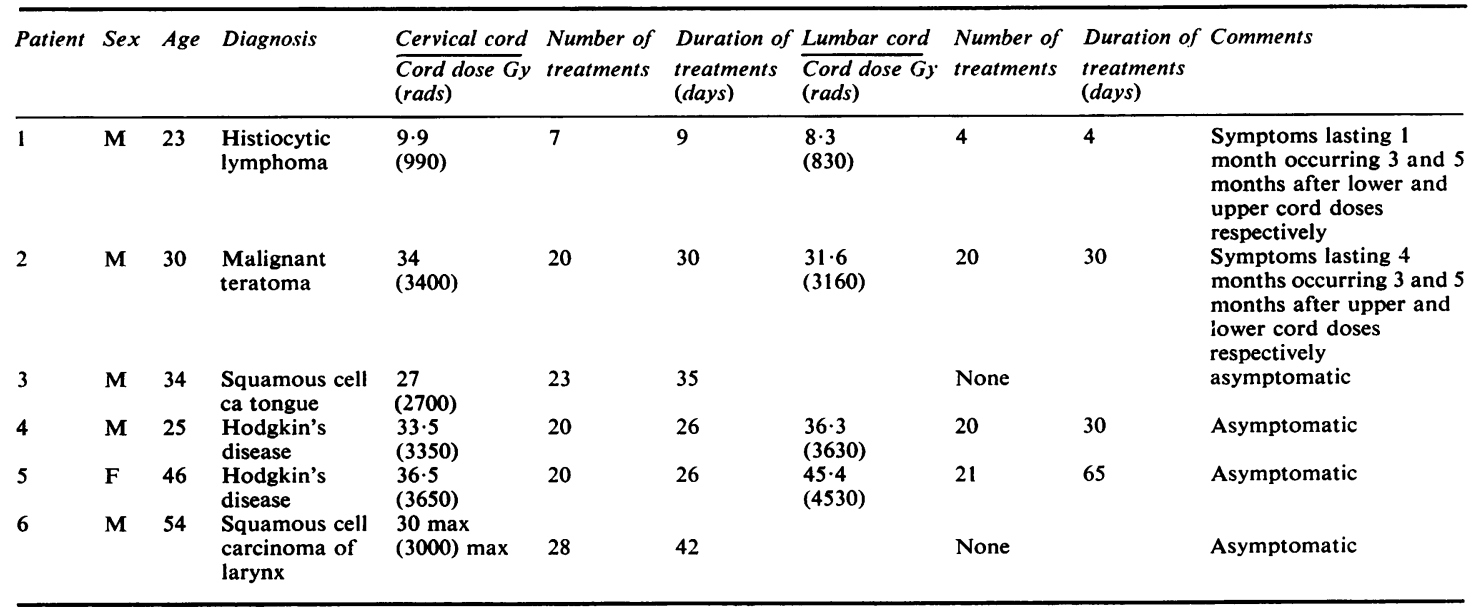

The radiotherapy patients were examined neurologically and the SEPs measured before and directly after treatment and again after an interval of about six months.

SEPs were recorded as described by Matthews et al. ${ }^{7}$ Stimuli at two per second were applied to the median nerve at the wrist at four times sensory threshold current. Records were taken from surface electrodes over Erb's point, between the sixth and seventh cervical spines $\left(C_{7}\right)$, rostral to the second cervical spine $\left(C_{2}\right)$ and over the hand area of the contralateral parietal region, using a common mid-frontal reference electrode. Three averages of 256 responses were taken on each side. The amplitude and the latency of the three major negative deflections, N9, N13 and N20 were measured.

A normal record is shown (fig 1). Wave N9, generated in the medial cord of the brachial plexus, was recorded over the mid-clavicular point. Wave N13, recorded over $\mathrm{C}_{2}$ and $\mathrm{C}_{7}$ is probably generated in the grey matter of the spinal cord or brainstem, no further rostral than the cuneate nucleus. ${ }^{89} \mathrm{~N} 20$, the first component of the cortical response, was recorded from the parietal hand area.

Normal values were obtained from 26 subjects with no neurological disease, and the latencies of N9, N13 and N20 correlated with arm length. Abnormality was defined as a value in excess of $2.5 \mathrm{SD}$ above the regression line of arm length plotted against latency for these controls.

\section{Results}

Although none of the six patients showed any abnormality on neurological examination at any stage, two did develop Lhermitte's symptom, consistent with the description of transient radiation myelopathy.

Patient 1 Five months after the completion of the upper cord exposure and three months after that of the lower, this patient developed intermittent tingling in the mid-dorsal and lumbar region radiating down the back of the thigh to the heels. This was provoked by either walking or by neck

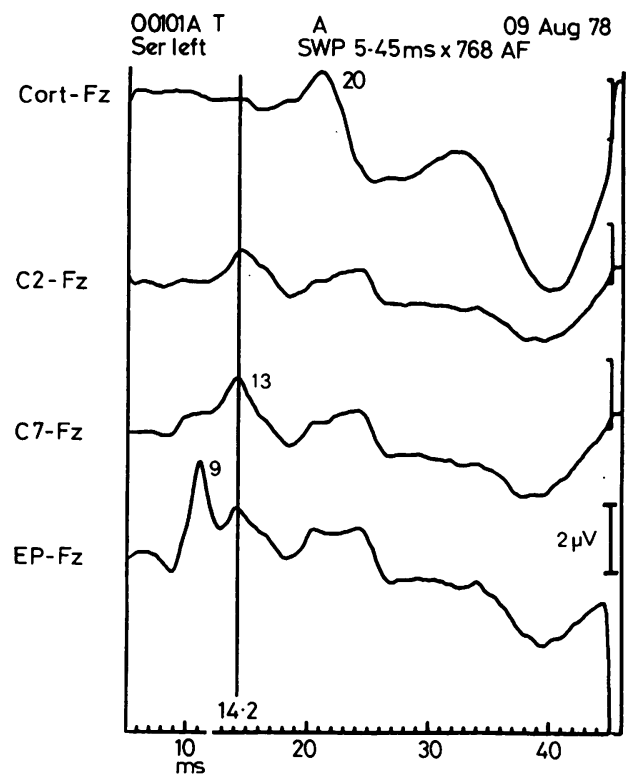

Fig 1 Normal cervical and cortical somatosensory evoked potentials (negative polarity upwards) with stimulation of the left median nerve at the wrist. Recording electrodes are over Erb's Point $(E P)$, the $C_{6}-C_{7}$ intervertebral space $\left(C_{7}\right)$, just rostral to the second cervical vertebra $\left(C_{2}\right)$ and the hand area of the right parietal cortex. There is a common midfrontal reference electrode $(F z)$. The main components are labelled 9,13, 20 and the cursor is on $N_{13}$. 




Fig 2 The $N_{9}-N_{13}$ latency interval for each side in the first record, prior to radiotherapy, is plotted against that in the final record after radiotherapy One result is overprinted (first record $3.7 \mathrm{~ms}$, final record $3.5 \mathrm{~ms}$ ). The upper limit of the normal $\mathrm{N}_{9}-$ $N_{13}$ latency for the shortest arm length in this series is $4 \cdot 2$ msec (mean $\left.+2 \frac{1}{2} S D\right)$. The points are clustered.

flexion and would pass off after ten minutes. The symptom resolved after one month and occurred between the second and third recordings.

Patient 2 Five months after lower and three months after upper cord exposure, this patient developed "shock-like" tingling of the buttocks and posterior thighs on flexing his neck, maintained flexion producing symptoms lasting two or three seconds. Occasionally the sensation would extend to the calves and soles of the feet. Resolution occurred in four months. Although improved, these symptoms were still present at the time of the third recording.

In the serial records no abnormality was found. On all occasions the amplitude and latencies of $\mathrm{N}_{9}$, $\mathrm{N}_{13}$ and $\mathrm{N}_{20}$ were within the normal range. Using the paired $t$ test no significant difference was found at the $5 \%$ level in the latencies of the major negative components or in the N9-N13 interval (fig 2) or N13-N20 interval between the three recordings.

\section{Discussion}

The origin of Lhermitte's symptom clearly must involve mechanical effects on the spinal cord. In the demyelinated cat dorsal root, increased mechanical irritability has been demonstrated, although this experimental lesion may have involved some axonal damage as well. ${ }^{10}$ To date however there is no direct evidence for such an effect in the spinal cord. The N9-N13 interval is believed to be a measure of the conduction time between the brachial plexus and the grey matter of the cervical cord and lower brainstem and reflects the conduction velocity in the dorsal column fibres. Our study has failed to demonstrate changes in this parameter likely to result from demyelination but we note that if experimental demyelinating lesions affect only a short segment of cord, slowing of conduction time may not be noticeable (McDonald and Smith, personal communication). Furthermore, production of symptoms is likely to be dependent not only on the size and number of lesions but also on their distribution, for lesions at sites of mechanical deformation of the cord may be more likely to give rise to symptoms.

Our electrophysiological study showed no detectable disturbance with cervical cord doses up to $36.5 \mathrm{~Gy}$ (3650 rad). The study of Mastaglia $e^{2} \mathrm{al}^{5}$ in which demyelination resulted from single doses of 1 to $60 \mathrm{~Gy}$ (100 to $6000 \mathrm{rad}$ ) is clearly difficult to compare directly with our investigation of patients receiving fractionated doses to the cervical cord.

We conclude that if spinal demyelination is responsible for transient radiation myelopathy, the lesions are likely to be short and accompanied by alteration in axon membrane properties causing increased sensitivity to mechanical distortion.

We thank Dr CJ Earl and Dr DG Small for their encouragement, and Professor A Jones and Professor WI McDonald for helpful discussion. We thank the consultant staff of the Myerstein Institute of Radiotherapy and Oncology, the Middlesex Hospital, for allowing the study of patients under their care, Dr B Planskoy for calculating the spinal cord doses and Julia Hampshire for recording the SEPs.

\section{References}

1 Boden G. Radiation myelitis of cervical spinal cord. Br J Radiol 1948; 21:464-9.

2 Jones A. Transient Radiation myelopathy $\mathrm{Br} J$ Radiol 1964; 37:727-44.

3 Innes JRM, Carsten A. A demyelinating or malacic-myelopathy and myodegeneration-delayed effect of localised $\mathrm{X}$-irradiation in experimental rats and monkeys. In Haley TJ, Snider RS, eds. Responses of the nervous system to ionising radiation. New York: Churchill 1962; 233-46.

4 Bailey OT, (1962). Basic problems in the histopathology of radiation of the central nervous system. In Haley TJ, and Snider RS, eds. Responses of the nervous system to ionising radiation. New York, Churchill, 1962; 165-189.

5 Mastaglia FL, McDonald WI, Watson JV, and Yogendran K. Effects of X-radiation on the spinal cord: an experimental study of the morphological changes in central nerve fibres Brain 1976; 99:101-22.

6 Small DG, Matthews WB, and Small M. The cervical somatosensory evoked potential (SEP) in 
the diagnosis of multiple sclerosis. $J$ Neurol $S c i$ 1977; 35:211-24.

7 Matthews WB, Beauchamp M, Small DG. Cervical somatosensory evoked responses in man. Nature 1974; 252:230-2.

8 Jones SJ. Short latency potentials recorded from the neck and scalp following median nerve stimulation in man. Electroencephalogr and Clin Neurophysiol 1977; 43:853-63.
9 El-Negamy E, Sedgwick EM. Properties of a spinal somatosensory evoked potential recorded in man. J Neurol Neurosurg Psychiatry 1978; 41: 762-8.

10 Howe JF, Loeser JD, Calvin WH. Mechanosensitivity of dorsal root ganglia and chronically injured axons: a physiological basis for the radicular pain of nerve root compression. Pain 1977; 3:25-41. 